\title{
BMJ Open Detection of peripheral and central sensitisation at acupoints in patients with unilateral shoulder pain in Beijing: a cross-sectional matched case- control study
}

Chao-Qun Yan, Shuai Zhang, Qian-Qian Li, Li-Wen Zhang, Xue-Rui Wang, Qing-Nan Fu, Guang-Xia Shi, Cun-Zhi Liu

To cite: Yan C-Q, Zhang $S$, Li Q-Q, et al. Detection of peripheral and central sensitisation at acupoints in patients with unilateral shoulder pain in Beijing: a cross-sectional matched case-control study. BMJ Open 2017;7:e014438. doi:10.1136/ bmjopen-2016-014438

- Prepublication history and additional material for this paper are available online. To view these files please visit the journal online (http://dx.doi.org/ 10.1136/ bmjopen-2016-014438).

Received 29 September 2016 Revised 10 April 2017 Accepted 26 April 2017

\section{(1) CrossMark}

The Department of Acupuncture and Moxibustion, Beijing Hospital of Traditional Chinese Medicine affiliated to Capital Medical University; Beijing Key Laboratory of Acupuncture Neuromodulation, Beijing, China

Correspondence to

Professor Cun-Zhi Liu;

Icz623780@126.com

\section{ABSTRACT}

Objective To investigate the pattern of experimental pain responses at acupoints in patients with unilateral shoulder pain.

Design A cross-sectional matched study.

Setting Acupuncture and Moxibustion Department, Beijing Hospital of Traditional Chinese Medicine Affiliated to Capital Medical University.

Participants Volunteer samples of 60 participants (30 patients with unilateral shoulder pain, 30 healthy controls). Interventions Not applicable.

Main outcome measures Pressure pain thresholds (PPTs) were measured at four acupoints-namely, Tianzong (SI 11), Jianliao (SJ 14), Jianyu (LI 15) and Jianzhen (SI 9), on the painful/non-painful side in patients with unilateral shoulder pain or healthy controls, respectively. The correlations between the Peripheral Sensitisation Index (PSI) and Central Sensitisation Index (CSI) were compared.

Results Analysis showed significantly lower PPT values at acupoints on the painful side compared with the nonpainful side in patients with shoulder pain $(p<0.025)$. Meanwhile, PPTs on the non-painful side of these patients were lower than those on the ipsilateral side of healthy controls $(p<0.025)$. No significant differences in PPT values were found between the non-acupoint of the painful/non-painful side in patients with shoulder pain and the ipsilateral side of healthy controls $(p>0.05)$. Additionally, it was observed that the pressure pain assessment acupoints have a strong association with PSI and CSI; three acupoints, in particular, SJ 14, LI 15 and SI 9, showed a correlation with PSI and CSI.

Conclusion The results suggest the presence of peripheral and central sensitisation at acupoints in participants with unilateral shoulder pain. There exists an obvious relationship among the three acupoints SJ 14, LI 15 and SI 9, which are usually chosen to treat shoulder pain. The results provide evidence for the selection of acupoints to treat shoulder pain by acupuncture.

\section{INTRODUCTION}

Acupuncture is one of the most widely used forms of complementary and alternative
Strengths and limitations of this study

- To our knowledge, this is the first study to support an alteration in both peripheral and central sensitisation at acupoints in patients with shoulder pain.

- The internal validity is increased by blinding the evaluator and participants.

- A limitation of this study is that pressure pain assessment was performed only at acupoints, which might be inadequate to describe the complexity of pain perception.

- This study was conducted in a single institution and consisted primarily of female subjects, so the external validity is unclear.

medicine. According to a survey conducted by the World Federation of Acupuncture and Moxibustion Societies in 2013 about 183 countries use acupuncture treatment. ${ }^{1}$ WHO reports that acupuncture treatment can be beneficial for more than 40 disorders. ${ }^{2}$ Pain is particularly amenable to acupuncture. Evidence in reviews demonstrates that acupuncture treatment can effectively reduce various types of clinical pain in multiple clinical trials. ${ }^{3-5}$ One meta-analysis reported that shoulder pain, low back pain, neck pain, osteoarthritis and chronic headache was significantly alleviated after acupuncture treatment. ${ }^{6}$ Shoulder pain is the third most common musculoskeletal disorder, with a prevalence ranging from $6.9 \%$ to $26 \%$ for point prevalence and increasing to $66.7 \%$ for lifetime prevalence in the general population. ${ }^{78}$ In our previous study, we showed that acupuncture can alleviate shoulder pain, particularly in particular acupoints (unpublished data).

Acupoints are special sites at precise locations and lie on 'meridians' without a physical 
structure. According to the theory of meridians in traditional Chinese medicine, it is well known that acupoints reflect disorders of visceral conditions. Many studies have observed that visceral lesions result in changed pain perception of some particular acupoints on the body surface. ${ }^{9}$ Ben et al assessed the phenomenon of experimental pain-sensitive points in patients with gastric ulcer or gastritis and found that some acupoints were more sensitive than other areas. ${ }^{10}$ Nevertheless, whether acupoints are more sensitive in patients with musculoskeletal pain has not been described in detail in previous studies.

Sensitisation is a nervous system phenomenon that can occur in conjunction with pain. ${ }^{11}$ When sensitisation is present, two types of hypersensitivity occur, including peripheral sensitisation and central sensitisation, which are important mechanisms in musculoskeletal pain. ${ }^{12}$ Peripheral sensitisation is defined as the broadening of nociception in pain perception during activities or movements, which typically would be not painful. ${ }^{11}$ Central sensitisation refers to an amplification of neural signalling within the central nervous system that elicits pain hypersensitivity. It reflects increased activity of pain facilitation pathways and malfunctioning of descending pain inhibitory pathways. ${ }^{13-17}$

We performed a multicentre, single blind, factorial randomised controlled clinical trial previously (Number Register: ISRCTN61861069). In that study, we found that the decrease in the pain threshold at related acupoints in patients with unilateral shoulder pain was widespread. Therefore, we chose some patients from the trial to detect whether acupoints are hypersensitive in musculoskeletal pain disease. We hypothesised that the patients with unilateral shoulder pain would present peripheral or central sensitisation at acupoints as shown by pressure pain threshold (PPT) detection, in comparison with healthy controls. Specifically, we suggested that acupoints on the painful side would have a lower PPT than that on the non-painful side of patients. Also, acupoints on the non-painful side of patients would be hypersensitive, as assessed by PPTs, with respect to the ipsilateral side of healthy controls. If the results confirm these hypotheses, then they provide evidence that acupoints exist
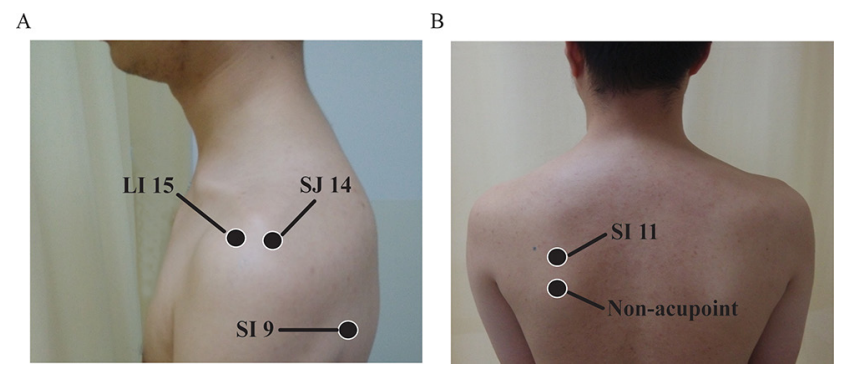

Figure 1 The pressure pain assessment sites in this study. (A) Locations of the pressure pain threshold (PPT) measurement at Jianliao (SJ 14), Jianyu (LI 15) and Jianzhen (SI 9). (B) Locations for the PPT measurement at Tianzong (SI 11) and a non-acupoint. for the peripheral sensitisation and central sensitisation phenomena in patients with unilateral shoulder pain.

\section{METHODS AND ANALYSIS \\ Study design}

This cross-sectional matched case-control study was conducted at the Department of Acupuncture and Moxibustion, Beijing Hospital of Traditional Chinese Medicine affiliated to Capital Medical University.

\section{Participants}

Clinical patients-Thirty patients with shoulder pain were chosen from a total of 164 patients with shoulder pain recruited from three centres to a randomised trial between January 2014 and September 2014. We enrolled the final 30 patients from 76 patients who had been screened at the Beijing Hospital of Traditional Chinese Medicine centre before any treatments were dispensed. The research ethical committee of Beijing Hospital of Traditional Chinese Medicine affiliated to Capital Medical University approved the trial (reference: 201315).

The inclusion criteria were presenting with unilateral shoulder pain for at least 6 weeks and up to 2 years; reporting the pain intensity as $>50 \mathrm{~mm}$ on a Visual Analogue Scale (VAS); being right hand dominant and not having received acupuncture or other analgesic treatments in the preceding month.

The exclusion criteria were as follows: pain in both shoulders; referred pain from the cervical spine; previous history of shoulder surgery, pectoral muscle pain, thoracic outlet syndrome, stroke or ipsilateral breast surgery; heart diseases and severe hypertension; osteoarthritis of the glenohumeral joint or systemic bone and joint disorder (rheumatoid arthritis); endocrine diseases such as hyperthyroidism; severe infection; undergoing current treatment involving analgesics and especially, major illness depression.

Healthy controls-The healthy controls were matched to the patients with shoulder pain individually. Each healthy control was matched for gender, age $( \pm 1$ year), ethnicity and dominant hand to one patient. Healthy controls were recruited from the community via posted flyers and general advertisements between May 2014 and September 2014. Healthy controls were eligible if they were not currently performing resistance exercise for the upper extremity. They were excluded based on the following criteria: had received acupuncture or other analgesic treatments in the preceding month; experiencing neck or shoulder pain; had a history of shoulder surgery or neurological impairments of the upper extremity; had a shoulder skin infection, had difficulty in understanding instructions and were currently taking any pain medication.

\section{Protocol}

All participants completed a number of questionnaires, including demographic data (age, sex, race, dominant hand) and psychological information, before informed 
Table 1 Characteristics of the patients with shoulder pain and healthy controls

\begin{tabular}{lll}
\hline Characteristics & Patients with shoulder pain $(\mathbf{n}=\mathbf{3 0})$ & Healthy controls $(\mathbf{n}=\mathbf{3 0})$ \\
\hline Age (years) & $50.60 \pm 12.19$ & $50.63 \pm 12.20$ \\
Sex (female), $\mathrm{n}(\%)$ & $19(63.3)$ & $19(63.3)$ \\
Race (number of Han), $\mathrm{n}(\%)$ & $30(100)$ & $30(100)$ \\
Pain duration (weeks) & $19.07 \pm 16.99$ & - \\
VAS (median, IQR) & $70(50-80)$ & - \\
BDI (median, IQR) & $0(0-0)$ & $0(0-0)$ \\
BMI & $25.27 \pm 4.17$ & $24.91 \pm 3.77$ \\
Normal (s23.9), $\mathrm{n}$ & 11 & 12 \\
Overweight $(24-27.9), \mathrm{n}$ & 12 & 14 \\
Obese $(\geq 28), \mathrm{n}$ & 7 & 4 \\
\hline
\end{tabular}

BDI, Beck Depression Inventory; BMI, Body Mass Index; VAS, Visual Analogue Scale.

consent was obtained. The patients were initially examined by a researcher who assessed compliance using the inclusion and exclusion criteria. The Beck Depression Inventory (BDI), a multiple choice self-reported inventory for measuring the severity of depression, was used. ${ }^{18}$ If the BDI outcome was $>4$, possible participants were excluded. ${ }^{19}$ Clinical pain intensity was assessed by a VAS, which consists of a $0-100 \mathrm{~mm}$ line, with 0 representing "no pain at all' and $100 \mathrm{~mm}$ representing 'the most intense pain imaginable'.

After completing the questionnaires, both shoulders of participants were exposed and the measurement sites were marked. The participants were not told which site was the non-acupoint. An expert acupuncturist was responsible for the procedure. Five points were marked in this study as shown in figure 1 and Supplementary appendix table 1. To assess the pain sensitivity of acupoints, four acupoints Jianliao (SJ 14), Jianyu (LI 15), Jianzhen (SI 9) and Tianzong (SI 11), were shown bilaterally by a circle drawn with a marker pen (figure $1 \mathrm{~A}, \mathrm{~B}$ ). To assess the pain sensitivity in the non-acupoint, a point $2 \mathrm{~cm}$ down from Tianzong (SI 11) was marked bilaterally in participants (figure 1B).

\section{Measurement of PPTs}

Both shoulders were exposed for examination by an operator. The participants were asked to take a prone position on the examination bed with a suitable pillow under the chest when Jianzhen (SI 9), Tianzong (SI 11) and the non-acupoint were measured. Then participants were required to sit on a chair with a researcher helping to keep the arm and shoulder in parallel when Jianliao (SJ 14) and Jianyu (LI 15) were measured.

PPT is widely used to evaluate the pain sensory threshold of patients with shoulder pain. ${ }^{20} \mathrm{~A}$ lower value of PPT indicates a decreased nociceptive threshold of pain perception. ${ }^{21}$ A handheld electronic pressure algometer (Wagner Instruments, Greenwich, Connecticut, USA) mounted with a $1 \mathrm{~cm}^{2}$ rubber tipped plunger was used in this study. The probe was held perpendicular to marked points bilaterally, and the pressure was increased at a rate of $30 \mathrm{kPa} / \mathrm{s}$, in order to avoid potential skin penetration. All participants were instructed to indicate when the pressure became painful; at that time the pressure was immediately stopped and the digits were recorded. PPT was calculated as the mean of three trials on each point. There was an approximately 2 min interval between repetitions. The operator was not told which side was the painful shoulder or whether the subject was a patient or a healthy controls. Additionally, the operator had no basic knowledge of acupoints and did not know whether the measuring sites were acupoints or not during testing.

\section{Data analysis}

The data were double entered with an adequate check in EpiData. SPSS 17.0 software (SPSS Inc, Chicago, Illinois, USA) was used for analysis. Discrete variables were summarised as frequencies and percentages. Distributed data were summarised using mean \pm SD or median and IQR. The distributed data were analysed using a parametric statistical test (paired t-test) if it agreed with normal distribution. Otherwise, the data were analysed using a non-parametric (Wilcoxon's signed rank test) statistical test. A Shapiro-Wilk test and observation of histograms and normal probability plots were used for all study variables to determine whether they followed a normal distribution. To adjust for multiple comparisons, an $\alpha$ level of 0.025 was used for all pairwise comparisons.

Peripheral and Central Sensitisation Indexes were used to determine whether pain sensitivity existed at the pressure pain assessment sites. Peripheral sensitisation referred to a patient's response for PPT on the painful side that fell below the 25 th centile compared with the non-painful side. ${ }^{22}$ The PPT value of the 25 th centile is determined by the average value of seventh and eighth lowest observations on the non-painful side. We examined a patient's proportional response for PPT on the painful side if it fell below the 25th centile. Each response PPT is considered for the Peripheral Sensitisation Index (PSI).

Central sensitisation indicates that a patient's response for the PPT on the non-painful side fell below the 25th centile (the average value of the seventh and eighth 

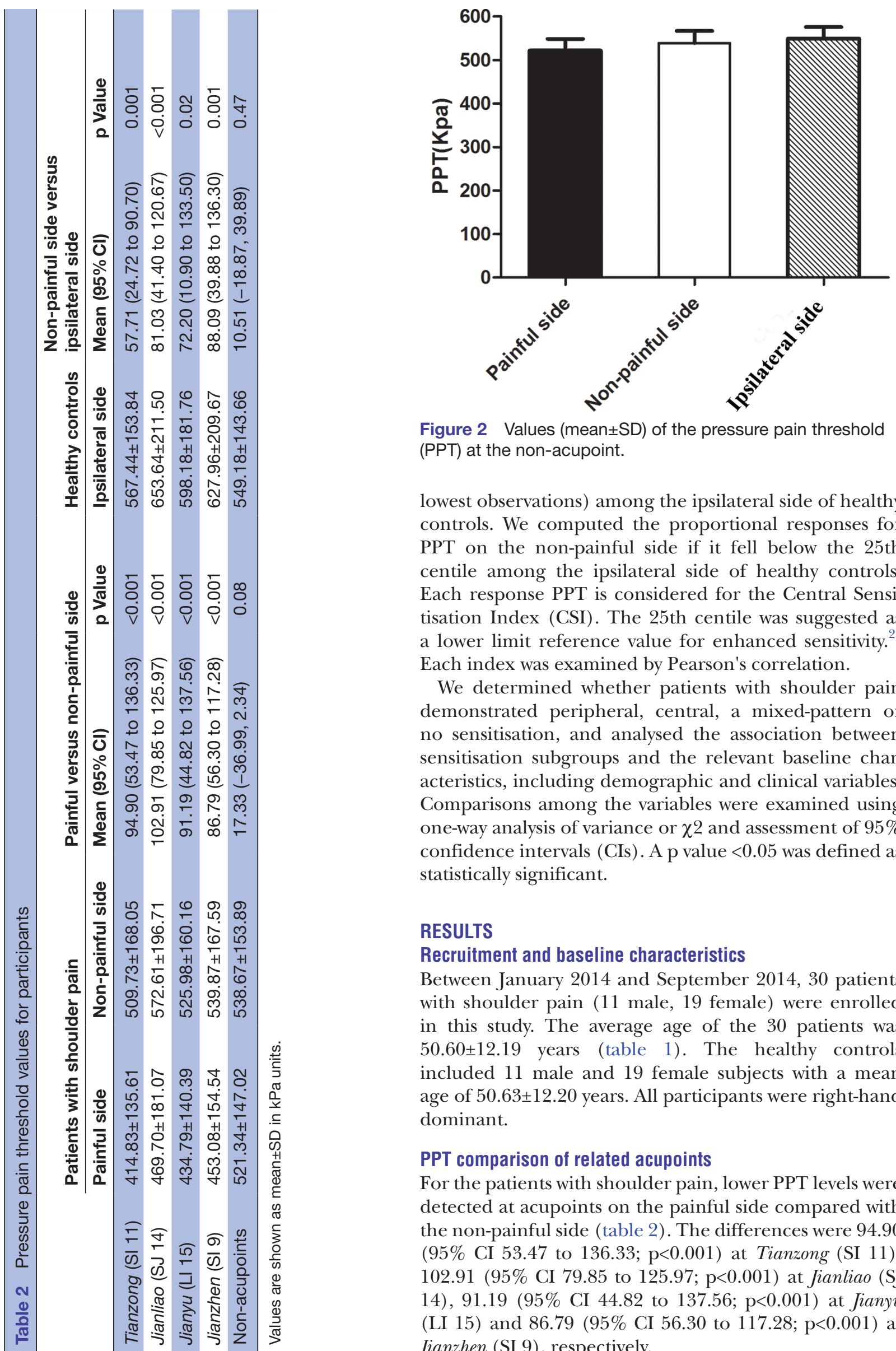

Figure 2 Values (mean $\pm S D$ ) of the pressure pain threshold (PPT) at the non-acupoint.

lowest observations) among the ipsilateral side of healthy controls. We computed the proportional responses for PPT on the non-painful side if it fell below the 25th centile among the ipsilateral side of healthy controls. Each response PPT is considered for the Central Sensitisation Index (CSI). The 25th centile was suggested as a lower limit reference value for enhanced sensitivity. ${ }^{23}$ Each index was examined by Pearson's correlation.

We determined whether patients with shoulder pain demonstrated peripheral, central, a mixed-pattern or no sensitisation, and analysed the association between sensitisation subgroups and the relevant baseline characteristics, including demographic and clinical variables. Comparisons among the variables were examined using one-way analysis of variance or $\chi 2$ and assessment of $95 \%$ confidence intervals (CIs). A p value $<0.05$ was defined as statistically significant.

\section{RESULTS}

\section{Recruitment and baseline characteristics}

Between January 2014 and September 2014, 30 patients with shoulder pain (11 male, 19 female) were enrolled in this study. The average age of the 30 patients was $50.60 \pm 12.19$ years (table 1 ). The healthy controls included 11 male and 19 female subjects with a mean age of $50.63 \pm 12.20$ years. All participants were right-hand dominant.

\section{PPT comparison of related acupoints}

For the patients with shoulder pain, lower PPT levels were detected at acupoints on the painful side compared with the non-painful side (table 2). The differences were 94.90 (95\% CI 53.47 to $136.33 ; \mathrm{p}<0.001)$ at Tianzong (SI 11 ), 102.91 (95\% CI 79.85 to $125.97 ; \mathrm{p}<0.001)$ at Jianliao (SJ 14), 91.19 (95\% CI 44.82 to $137.56 ; \mathrm{p}<0.001)$ at Jianyu (LI 15) and 86.79 (95\% CI 56.30 to $117.28 ; \mathrm{p}<0.001$ ) at Jianzhen (SI 9), respectively. 
The PPT value difference between the non-painful side of the patients and the ipsilateral side of healthy controls was also compared. PPTs at the acupoints on non-painful side of the patients were lower than those on the ipsilateral side of healthy controls. The differences were 57.71 (95\% CI 24.72 to $90.70 ; \mathrm{p}=0.001$ ) at Tianzong (SI 11), $81.03(95 \%$ CI 41.40 to $120.67 ; \mathrm{p}<0.001)$ at Jianliao (SJ 14), 72.20 (95\% CI 10.90 to $133.50 ; \mathrm{p}=0.02$ ) at Jianyu (LI $15)$ and 88.09 (95\% CI 39.88 to $136.30 ; \mathrm{p}=0.001)$ at Jianzhen (SI 9), respectively.

\section{PPT comparison of non-acupoint}

Figure 2 shows PPT at the non-acupoint. For patients, the analysis revealed no marked difference in PPT value (17.33 (95\% CI -36.99 to $2.34 ; \mathrm{p}=0.08)$ on the painful side compared with the non-painful side. Similarly, no significant difference of PPT level 10.51 was found between the non-painful side of the patients and the ipsilateral side of healthy controls $(95 \% \mathrm{CI}-18.87$ to 39.89; $\mathrm{p}=0.47$ ),

\section{Peripheral sensitisation index}

All measured acupoints demonstrated side-to-side difference in the patients. They were used to compute a peripheral sensitisation index (PSI). PPT values at acupoints on the painful side below the 25th centile of the non-painful side indicated peripheral sensitisation. The proportion of the patients with peripheral sensitisation was $77 \%$ at Tianzong (SI 11), 37\% at Jianliao (SJ 14), $43 \%$ at Jianyu (LI 15) and 60\% at Jianzhen (SI 9) (table 3). Significant correlation (Supplementary appendix table 2) was observed in PSI among Jianliao (SJ 14), Jianyu (LI 15) and Jianzhen (SI 9) $(p<0.01)$. There was no significant association between PSI and other baseline variables (p>0.05) (Supplementary appendix table 3).

\section{Central Sensitisation Index}

The four measured acupoints demonstrated a difference between the non-painful side of patients and the ipsilateral side of healthy controls. Therefore, they were used to compute the central sensitisation index (CSI). The proportion of the patients with central sensitisation was $43 \%$ at Tianzong (SI 11), 57\% at Jianliao (SJ 14), 63\% at Jianyu (LI 15) and 60\% at Jianzhen (SI 9) (table 3). A distinct and significant association (Supplementary appendix table 4) was observed between Jianliao (SJ 14)

\begin{tabular}{|c|c|c|c|}
\hline Sites & PSI & CSI & PSI+CSI \\
\hline Tianzong (SI 11) & $23(77)$ & $13(43)$ & $13(43)$ \\
\hline Jianliao (SJ 14) & $11(37)$ & $17(57)$ & $9(30)$ \\
\hline Jianyu (LI 15) & $13(43)$ & $19(63)$ & $12(40)$ \\
\hline Jianzhen (SI 9) & $18(60)$ & $18(60)$ & $15(50)$ \\
\hline
\end{tabular}

Values are individual counts (percentages). and Jianyu (LI 15) $(\mathrm{p}<0.01)$, Jianyu (LI 15) and Jianzhen (SI 9) $(\mathrm{p}<0.01)$ in CSI. Supplementary appendix table 5 showed no statistical significance correlations between CSI and relevant baseline characteristics ( $p>0.05)$.

\section{Comparison of peripheral and central sensitisation index}

The frequencies of the patients who had both PSI and CSI were $43 \%$ at Tianzong (SI 11), 30\% at Jianliao (SJ 14), $40 \%$ at Jianyu (LI 15) and 50\% at Jianzhen (SI 9), respectively (table 3). Significant correlation was observed between PSI and CSI in measured acupoints $(\mathrm{p}<0.05)$ (Supplementary appendix table 6 ). Table 4 shows that association between subgroups and gender approached statistical significance at the assessment acupoints $(\mathrm{p}<0.001)$. No significant association was observed between subgroups and other baseline variables $(\mathrm{p}>0.05)$.

\section{DISCUSSION}

Our results are in agreement with a peripherally sensitised state at acupoints which is determined by a difference of PPT values between the sides in patients with unilateral shoulder pain. Central sensitisation at acupoints was conducted by comparing pressure sensitivity in patients with that in age- and sex-matched healthy controls. No clear difference in PPT values was found at the non-acupoint among the painful side, non-painful side and ipsilateral side. To advance this line of research, association between peripheral and central sensitisation at acupoints was examined. We found that the patients had a significant association between peripheral and central sensitisation at measured acupoints. This finding demonstrates that there are two patterns of sensitisation at acupoints. In addition, three acupoints Jianliao (SJ 14), Jianyu (LI 15) and Jianzhen (SI 9) that are normally used for treating shoulder pain correlated with CSI and PSI.

Previous investigations reported that splanchnic diseases can induce mechanical hyperalgesia on the corresponding acupoints when pressed. ${ }^{24}$ Acupoints became activated or sensitised in pathological conditions. This phenomenon is called 'acupoint sensitisation'. At some acupoints there appeared to be hypersensitivity towards temperature (heat sensitisation) or pain threshold (pain-sensitisation) under visceral pain. ${ }^{95}$ But unlike those studies, we examined acupoints in patients with musculoskeletal pain in this study.

Central sensitisation is challenging clinically, since no standard assessment exists. Some studies recommended the use of various modalities for pain sensitivity at local and distal locations. ${ }^{21}{ }^{26}$ However, other research shows reduced PPTs at the painful and non-painful shoulder, but not at the muscle tibialis anterior. ${ }^{27-29}$ According to the 'Criteria for the Classification of Central Sensitisation Pain', patients with diffuse pain distribution, allodynia, and hyperalgesia are more likely to present with central sensitisation. One of the patterns of pain distribution is that patients have bilateral pain/mirror pain. ${ }^{17}$ In patients with shoulder pain, the increased sensitivity to mechanical 


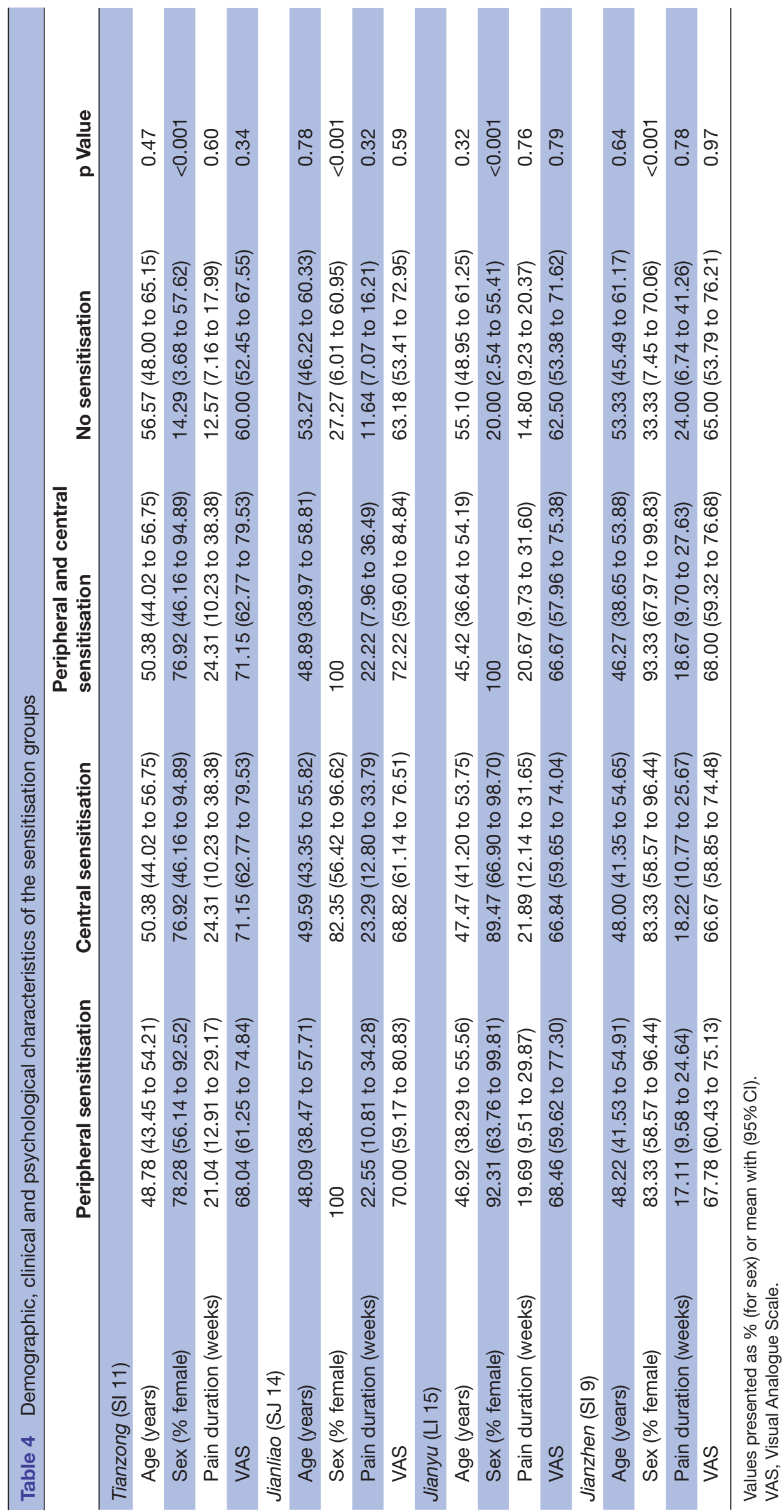


input in the contralateral shoulder would be interpreted as central sensitisation. ${ }^{11}$ A large number of studies define central sensitisation as pain sensitivity at local and distal locations. We chose bilateral pain to define central sensitisation, unlike earlier studies, and to determine whether there existed patterns of experimental pain responses at shoulder acupoints.

Collectively, the findings of the study support the alteration in both peripheral and central sensitisation at acupoints in patients with musculoskeletal pain. We determined whether peripheral and central sensitisation were more likely to occur together or alone. Table 3 shows that 19 patients had central sensitisation and 13 patients had peripheral sensitisation in Jianyu (LI 15). A previous study showed that long-term peripheral sensitisation can lead to central nervous system changes, resulting in central sensitisation. ${ }^{11}$ However, six patients had central sensitisation only, without peripheral sensitisation. This result indicates that peripheral sensitisation is not a prerequisite for the presence of central sensitisation at acupoints. In PSI or CSI, a strong association was found at three acupoints-namely, Jianliao (SJ 14), Jianyu (LI 15) and Jianzhen (SI 9). Jianliao (SJ 14), Jianyu (LI 15) and Jianzhen (SI 9) are frequently chosen to treat shoulder pain in clinical practice, and have been called the 'shoulder three acupoints' by acupuncturists. ${ }^{3031}$ They are highly refined acupoints used to treat shoulder pain in the clinic, and have been proved to effectively regulate muscle strength and tension of the shoulder joint. ${ }^{32}$ The strong association among Jianliao (SJ 14), Jianyu (LI 15) and Jianzhen (SI 9) is consistent with the concept of traditional Chinese medicine.

To our knowledge, this is the first study to research peripheral and central sensitisation at acupoints in patients with shoulder pain. One of the advantages of the study is that the measured acupoints and non-acupoint were marked by an acupuncturist with 24 years of experience in clinical acupuncture treatment. The evaluator who measured PPTs also had extensive experience with using an algometer and had no basic knowledge of acupoints. The internal validity was increased by blinding the evaluator, who did not know whether or not the measured sites were acupoints or whether the test participant was a patient or a healthy control. The participants were asked to take different positions when different acupoints were measured. For example, the participants were required take a prone position on the examination bed with a suitable pillow under the chest and the arms close to the body when Jianzhen (SI 9) was measured as this increases the reliability of testing PPT over a soft area.

Specifically, the results indicate that peripheral and central sensitisation at acupoints is not relevant to pain duration. Moreover, there is no obvious evidence that the pattern of sensitisation is related to the degree of severity of the clinical condition as measured by a VAS. These changes are consistent with the idea of traditional Chinese medicine that acupoints are the crucial reflex points of body lesions under pathological circumstances.
Hyperalgesia and skin sensitisation can occur at the corresponding acupoints in the presence of some diseases. ${ }^{33}$ Morphological structure studies have reported that the nervous system and blood vessels might have a close relationship with acupoints. ${ }^{34-36}$ For example, abundant microvessels existed at the acupoints of Zhongii (RN3) and Zusanli (ST36) in contrast to the surrounding tissues. ${ }^{37}$ The acupoints also have a high density of nerve endings including A- and C-afferent fibres. ${ }^{38}{ }^{39}$ Those characteristics of higher concentration of neural, vascular elements and mast cells could make pain perception more sensitive, and might contribute to peripheral sensitivity.

To confirm the specificity of acupoints, we selected a non-acupoint in the infraspinatus muscle. No significant difference was found in PPT values among the painful/ non-painful side of patients and the ipsilateral side of healthy controls. The finding proved that acupoints are the specific reflex points that respond to the presence of musculoskeletal pain. Our study provides evidence that there is an association between sensitisation of acupoints and gender. Pain difference in men and women has been increasingly studied in recent years, and lower PPT values in women than those in men were found both in healthy subjects and in clinical patients. ${ }^{40}$ But, the significant difference we found might be due to the fact that the majority of patients in the study $(63.3 \%)$ were female.

This study has some limitations. First, pain perception is multidimensional. PPT measurements are a mechanical and standardised stimulation and are inadequate to describe the complexity of pain perception. A multimodal approach should be used to provide details of the pain system in both normal and pathophysiological situations, such as different stimulus modalities and quantitative assessment of various pain mechanisms. Second, the non-acupoint was chosen as $2 \mathrm{~cm}$ down from Tianzong (SI 11) because the shoulder blade is relatively flat and may reduce measurement errors between acupoints and non-acupoints. In a clinical trial published recently, the distance was one cun $(2-3 \mathrm{~cm})$ between the non-acupoints and acupoints, and clinical outcome showed that treatment of acupoints provided greater alleviation of symptoms than treatment of non-acupoints. ${ }^{41}$ In some studies including measurement of PPT the measured sites are adjacent. ${ }^{20}{ }^{42}$ In our study the distance between the non-acupoint and Tianzong (SI 11) was $2 \mathrm{~cm}$, which is acceptable. To reduce the stimulation effect, there is an approximately $2 \mathrm{~min}$ interval between the repetitions. The probability of stimulation by an acupoint that is too close is low but cannot be ruled out completely. In addition, ashi points, also called reflexing points or tender spots, are temporary acupoints, which are dissimilar from acupoints of the 14 meridians or extraordinary points. In general, ashi points have no specific names and definite locations, and will vanish after recovery from the disease. The aim of our study was to investigate the pattern of experimental pain responses at acupoints, which have specific names and definite locations and therefore the ashi points were not taken into account in this study. This 
study was conducted in a single institution with a small sample size, so the external validity is unclear. Finally, this was a cross-sectional study, and it is not known whether the sensitisation experienced at acupoints changes as the disease progresses. Further studies are needed to confirm the phenomena observed here.

\section{CONCLUSIONS}

In conclusion, mixed sensitisation patterns occur at acupoints in patients with unilateral shoulder pain and a strong correlation among Jianliao (SJ 14), Jianyu (LI 15) and Jianzhen (SI 9). Future research using a multimodal pain approach should be conducted, such as suprathreshold heat pain response, to determine various sensitivity mechanisms.

Contributors Conceived and designed the experiments: Q-QL, C-ZL. Performed the experiments: G-XS, Q-NF, C-QY, Q-QL. Analysed the data: L-WZ, X-RW. Wrote the paper: C-QY, SZ. All authors approved the final manuscript.

Funding This work was supported by Beijing Municipal Administration of Hospitals Clinical Medicine Development of Special Funding Support grant number XMLX201607 and ZYLX201412.

Competing interests None declared.

Patient consent Obtained.

Ethics approval The Research Ethical Committee of Beijing Hospital of Traditional Chinese Medicine Affiliated to Capital Medical University.

Provenance and peer review Not commissioned; externally peer reviewed.

Data sharing statement No material in this paper has been published or is under consideration elsewhere.

Open Access This is an Open Access article distributed in accordance with the Creative Commons Attribution Non Commercial (CC BY-NC 4.0) license, which permits others to distribute, remix, adapt, build upon this work non-commercially, and license their derivative works on different terms, provided the original work is properly cited and the use is non-commercial. See: http://creativecommons.org/ licenses/by-nc/4.0/

(C) Article author(s) (or their employer(s) unless otherwise stated in the text of the article) 2017. All rights reserved. No commercial use is permitted unless otherwise expressly granted.

\section{REFERENCES}

1. WHO Traditional Medicine Strategy: 2014-2023. ISBN: 97892 41506090 (2013). http://www.who.int/edicines/publications/ traditional/trm_strategy14_23/en/.

2. http://apps.who.int/medicinedocs/pdf/s4926e/s4926e.pdf

3. De Kooning M, Tobbackx Y, Meeus M, et al. Acupuncture-analgesia following a single treatment session in chronic whiplash is unrelated to autonomic nervous system changes: a randomized cross-over trial. Pain Physician 2015;18:527-36.

4. MacPherson $\mathrm{H}$, Tilbrook $\mathrm{H}$, Richmond $\mathrm{S}$, et al. Alexander technique lessons or acupuncture sessions for persons with chronic neck pain: a randomized trial. Ann Intern Med 2015;163:653-62.

5. Pfister DG, Cassileth BR, Deng GE, et al. Acupuncture for pain and dysfunction after neck dissection: results of a randomized controlled trial. J Clin Oncol 2010;28:2565-70.

6. Vickers AJ, Cronin AM, Maschino AC, et al. Acupuncture for chronic pain: individual patient data meta-analysis. Arch Intern Med 2012;172:1444-53.

7. Luime JJ, Koes BW, Hendriksen IJ, et al. Prevalence and incidence of shoulder pain in the general population; a systematic review. Scand J Rheumatol 2004;33:73-81.

8. Luime JJ, Koes BW, Miedem HS, et al. High incidence and recurrence of shoulder and neck pain in nursing home employees was demonstrated during a 2-year follow-up. J Clin Epidemiol 2005;58:407-13.
9. Rong PJ, Li S, Ben H, et al. Peripheral and spinal mechanisms of acupoint sensitization phenomenon. Evid Based Complement Alternat Med 2013;2013:1-6.

10. Ben H, Li L, Rong P-J, et al. Observation of pain-sensitive points along the meridians in patients with gastric ulcer or gastritis. Evid Based Complement Alternat Med 2012;2012:1-7.

11. Borstad J, Woeste C. The role of sensitization in musculoskeletal shoulder pain. Braz J Phys Ther 2015;19:251-7.

12. Arendt-Nielsen L, Fernández-de-Las-Peñas $C$, Graven-Nielsen $T$. Basic aspects of musculoskeletal pain: from acute to chronic pain. $J$ Man Manip Ther 2011;19:186-93.

13. Woolf CJ. Central sensitization: implications for the diagnosis and treatment of pain. Pain 2011;152:S2-S15.

14. Nijs J, Van Houdenhove B, Oostendorp RA. Recognition of central sensitization in patients with musculoskeletal pain: application of pain neurophysiology in manual therapy practice. Man Ther 2010;15:135-41.

15. Staud R, Craggs JG, Robinson ME, et al. Brain activity related to temporal summation of $\mathrm{C}$-fiber evoked pain. Pain 2007;129:130-42.

16. Meeus M, Nijs J. Central sensitization: a biopsychosocial explanation for chronic widespread pain in patients with fibromyalgia and chronic fatigue syndrome. Clin Rheumatol 2007;26:465-73.

17. Nijs J, Torres-Cueco R, van Wilgen CP, et al. Applying modern pain neuroscience in clinical practice: criteria for the classification of central sensitization pain. Pain Physician 2014;17:447-57.

18. Smarr KL, Keefer AL. Measures of depression and depressive symptoms: Beck Depression Inventory-II (BDI-II), Center for Epidemiologic Studies Depression Scale (CES-D), Geriatric Depression Scale (GDS), Hospital Anxiety and Depression Scale (HADS), and Patient Health Questionnaire-9 (PHQ-9). Arthritis Care Res 2011;63:S454-S466.

19. Zheng HB, Zheng YP. The application of Beck Depression Inventory in depression patients. Chinese J Nerv Ment Dis 1987;13:236.

20. Ge HY, Fernández-de-Las-Peñas C, Madeleine P, et al. Topographical mapping and mechanical pain sensitivity of myofascial trigger points in the infraspinatus muscle. Eur J Pain 2008;12:859-65.

21. Paul TM, Soo Hoo J, Chae J, et al. Central hypersensitivity in patients with subacromial impingement syndrome. Arch Phys Med Rehabil 2012;93:2206-9.

22. Coronado RA, Simon CB, Valencia C, et al. Experimental pain responses support peripheral and central sensitization in patients with unilateral shoulder pain. Clin J Pain 2014;30:143-51.

23. Neziri AY, Scaramozzino P, Andersen OK, et al. Reference values of mechanical and thermal pain tests in a pain-free population. Eur $J$ Pain 2011;15:376-83.

24. Chae Y, Kim HY, Lee HJ, et al. The alteration of pain sensitivity at disease-specific acupuncture points in premenstrual syndrome. $J$ Physiol Sci 2007;57:115-9.

25. Peles S, Miranda A, Shaker R, et al. Acute nociceptive somatic stimulus sensitizes neurones in the spinal cord to colonic distension in the rat. J Physiol 2004;560:291-302.

26. Nijs J, Van Houdenhove B, Oostendorp RA. Recognition of central sensitization in patients with musculoskeletal pain: application of pain neurophysiology in manual therapy practice. Man Ther 2010;15:135-41.

27. Ge HY, Fernández-de-las-Peñas C, Arendt-Nielsen L. Sympathetic facilitation of hyperalgesia evoked from myofascial tender and trigger points in patients with unilateral shoulder pain. Clin Neurophysiol 2006;117:1545-50.

28. Alburquerque-Sendín F, Camargo PR, Vieira A, et al. Bilateral myofascial trigger points and pressure pain thresholds in the shoulder muscles in patients with unilateral shoulder impingement syndrome: a blinded, controlled study. Clin j Pain 2013;29:478-86.

29. Gwilym SE, Oag HC, Tracey I, et al. Evidence that central sensitisation is present in patients with shoulder impingement syndrome and influences the outcome after surgery. J Bone Joint Surg Br 2011;93:498-502.

30. Chen $L$. [A new thinking of acupuncture and moxibustion treatment of shoulder pain after hemiplegia]. Zhongguo Zhen Jiu 2006;26:669-71.

31. Li N, Tian FW, Wang CW, et al. [Double-center randomized controlled trial on post-stroke shoulder pain treated by electroacupuncture combined with tuina]. Zhongguo Zhen Jiu 2012;32:101-5.

32. Chen MY, Pu QQ, Liu SY, et al. [Efficacy comparison of different stimulation therapies for periarthritis of shoulder]. Zhongguo Zhen Jiu 2013;33:109-12. 
33. Li L, Yu L, Rong P, et al. Visceral nociceptive afferent facilitates reaction of subnucleus reticularis dorsalis to acupoint stimulation in rats. Evid Based Complement Alternat Med 2013;2013:1-7.

34. Kuo TC, Lin CW, Ho FM. The soreness and numbness effect of acupuncture on skin blood flow. Am J Chin Med 2004;32:117-29.

35. Zhang D, Yan X, Zhang X, et al. Synchrotron radiation phasecontrast X-ray CT imaging of acupuncture points. Anal Bioanal Chem 2011;401:803-8.

36. Wick F, Wick N, Wick MC. Morphological analysis of human acupuncture points through immunohistochemistry. Am J Phys Med Rehabil 2007;86:7-11.

37. Oh DS, Kang W, Choi SM, et al. Effect of acupuncture for gastrointestinal activity differs depending on the pathophysiological condition. Acupunct Med 2011;29:316-7.
38. Li AH, Zhang JM, Xie YK. Human acupuncture points mapped in rats are associated with excitable muscle/skin-nerve complexes with enriched nerve endings. Brain Res 2004;1012:154-9.

39. Zhu B, Xu WD, Rong PJ, et al. A C-fiber reflex inhibition induced by electroacupuncture with different intensities applied at homotopic and heterotopic acupoints in rats selectively destructive effects on myelinated and unmyelinated afferent fibers. Brain Res 2004;1011:228-37.

40. Tschugg A, Löscher WN, Hartmann S, et al. Gender influences radicular pain perception in patients with lumbar disc herniation. $J$ Womens Health 2015;24:771-6.

41. Liu Z, Yan S, Wu J, et al. Acupuncture for chronic severe functional constipation: a randomized trial. Ann Intern Med 2016;12;165:761-9.

42. Binderup AT, Arendt-Nielsen L, Madeleine P. Pressure pain threshold mapping of the trapezius muscle reveals heterogeneity in the distribution of muscular hyperalgesia after eccentric exercise. Eur J Pain 2010;14:705-12. 